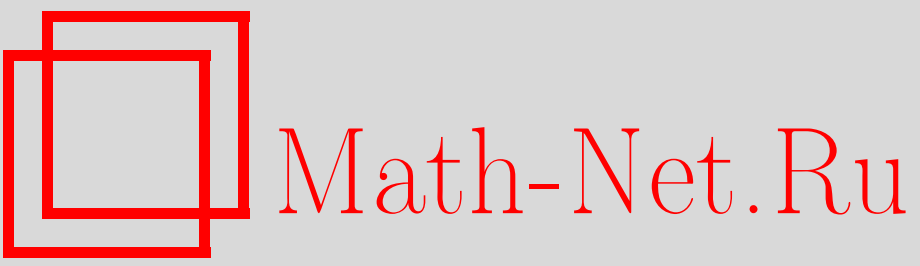

С. С. Мамонов, И. В. Ионова, А. О. Харламова, Кривизна циклов фазовых систем с мультистабильностью, Итоги науки и техн. Сер. Соврем. мат. и ее прил. Темат. обз., 2020, том 186, 83-90

DOI: https://doi.org/10.36535/0233-6723-2020-186-83-90

Использование Общероссийского математического портала Math-Net.Ru подразумевает, что вы прочитали и согласны с пользовательским соглашением

http://www . mathnet.ru/rus/agreement

Параметры загрузки:

IP : 54.166 .219 .16

26 апреля 2023 г., 16:47:36 


\title{
КРИВИЗНА ЦИКЛОВ ФАЗОВЫХ СИСТЕМ С МУЛЬТИСТАБИЛЬНОСТЬЮ
}

\author{
(c) 2020 г. $\quad$ С. С. МАМОНОВ, И. В. ИОНОВА, А. О. ХАРЛАМОВА
}

\begin{abstract}
АннотАция. В работе рассматривается система дифференциальных уравнений, описывающая математическую модель системы фазовой автоподстройки с запаздыванием. В работе вводится понятие кривизны цикла, которое применяется для проведения сравнительного анализа близости циклов нефазовой и фазовой систем.
\end{abstract}

Ключевые слова: система дифференциальных уравнений, колебательный цикл, фазовая система, кривизна цикла, вращение векторного поля.

\section{CURVATURE OF CYCLES OF PHASE SYSTEMS WITH MULTISTABILITY}

\author{
(c) 2020 S. S. MAMONOV, I. V. IONOVA, A. O. KHARLAMOVA
}

\begin{abstract}
In this paper, we consider a system of differential equations describing a mathematical model of a phase-locked loop with a delay. We introduce the concept of the curvature of a cycle, which is applied to analyzing the proximity of cycles of nonphase and phase systems.
\end{abstract}

Keywords and phrases: system of differential equations, oscillatory cycle, phase system, curvature of cycle, rotation of a vector field.

AMS Subject Classification: 34K05, 34K13, 34K60

1. Введение. Рассматривается система дифференциальных уравнений $[4,6,7]$

$$
\dot{x}=A x+b \varphi(\sigma), \quad \dot{\sigma}=c^{T} x+\rho_{1} \varphi(\sigma)+\rho_{0}(1-u) \varphi(\sigma)-\alpha_{1} u \sigma,
$$

где $x, b, c \in \mathbb{R}^{2}, u \in[0 ; 1], \alpha_{1}, \rho_{1}, \rho_{0} \in \mathbb{R}, \varphi(\sigma)-\Delta$-периодическая функция. При $u=0$ система (1) становится фазовой системой

$$
\dot{x}=A x+b \varphi(\sigma), \quad \dot{\sigma}=c^{T} x+\left(\rho_{1}+\rho_{0}\right) \varphi(\sigma) .
$$

Для систем дифференциальных уравнений актуальным является вопрос реализации бифуркационных процессов, определение нелинейных колебаний и их анализ [1-6]. Система уравнений (2) описывает динамику системы фазовой автоподстройки с запаздыванием в звене фильтра нижних частот в фазовой цепи управления $[2,4-6]$. Запаздывание в системе автоподстройки определяет параметр $\rho=\rho_{1}+\rho_{0}>0$, при отсутствии запаздывания выполняется равенство $\rho=0$.

Пусть функция $z(t)=\operatorname{colon}\left(x_{1}(t), x_{2}(t), \sigma(t)\right)$ определяет цикл системы $(1)$, тогда под кривизной цикла будем понимать величину

$$
K(t)=\frac{\sqrt{\left[z^{\prime}(t) \times z^{\prime \prime}(t)\right]^{2}}}{\left(z^{\prime 2}(t)\right)^{3 / 2}},
$$


где $z^{\prime}(t)=\operatorname{colon}\left(x_{1}^{\prime}(t), x_{2}^{\prime}(t), \sigma^{\prime}(t)\right), z^{\prime 2}=z^{\prime T} \cdot z^{\prime}, z^{\prime \prime}(t)=\operatorname{colon}\left(x_{1}^{\prime \prime}(t), x_{2}^{\prime \prime}(t), \sigma^{\prime \prime}(t)\right)$, а символ «Х» обозначает векторное произведение. В силу (3) кривизна цикла системы (1) удовлетворяет соотношению

$$
K(t)=\frac{\sqrt{\left(x_{1}^{\prime} x_{2}^{\prime \prime}-x_{1}^{\prime \prime} x_{2}^{\prime}\right)^{2}+\left(x_{2}^{\prime 2} \sigma^{\prime \prime}-x_{2}^{\prime \prime} \sigma^{\prime}\right)^{2}+\left(\sigma^{\prime} x_{1}^{\prime \prime}-\sigma^{\prime \prime} x_{1}^{\prime}\right)^{2}}}{{\left(x_{1}^{\prime}{ }^{2}+x_{2}^{\prime 2}+{\sigma^{\prime}}^{2}\right)^{3 / 2}}^{2}} .
$$

Системы (1) и (2) рассматривались в работах [4,6,7]. Для этих систем аналитическими методами были найдены начальные условия устойчивых и неустойчивых колебательных циклов, определены условия реализации в таких системах режимов мультистабильности, предложены численные методы определения близости циклов фазовой и нефазовой систем на основе их метрических характеристик, а также с использованием их кривизны.

В данной работе предлагается рассмотреть зависимость кривизны циклов от координат фазовой и нефазовой систем и использовать кривизну циклов для анализа мультистабильности.

2. Основные результаты. Сформулируем основные результаты работы. Рассмотрим систему дифференциальных уравнений (1), где

$$
\begin{gathered}
A=\left(\begin{array}{cc}
-\alpha_{1} & -\beta_{1} \\
1 & 0
\end{array}\right), b=\left(\begin{array}{c}
-\nu_{1} \\
-\Gamma
\end{array}\right), c=\left(\begin{array}{l}
0 \\
1
\end{array}\right), \\
\alpha_{1}>0, \beta_{1}>0, \varphi(\sigma)=-\gamma+\sin (\sigma+\arcsin \gamma), \varphi(0)=0,
\end{gathered}
$$

найдем $c^{T} b=-\Gamma, c^{T} A=l^{T}, l^{T} b=-\nu_{1}<0, \nu=-\nu_{1}, \operatorname{rang}\|c, l\|=2, l^{T} A=-\alpha_{1} l^{T}-\beta_{1} c^{T}$, $c^{T} A^{-1} b \neq 0, \rho=\rho_{1}+\rho_{0}>0$. Систему (1) запишем в виде

$$
\left\{\begin{array}{l}
\dot{x}_{1}=-\alpha_{1} x_{1}-\beta_{1} x_{2}+\nu \varphi(\sigma) \\
\dot{x}_{2}=x_{1}-\Gamma \varphi(\sigma) \\
\dot{\sigma}=x_{2}+\rho_{1} \varphi(\sigma)+\rho_{0}(1-u) \varphi(\sigma)-\alpha_{1} u \sigma
\end{array}\right.
$$

Найдем вторую производную в силу системы (5)

$$
\left\{\begin{array}{l}
x_{1}^{\prime \prime}=-\alpha_{1} x_{1}^{\prime}-\beta_{1} x_{2}^{\prime}+\nu \varphi_{\sigma}^{\prime}(\sigma) \sigma^{\prime}, \\
x_{2}^{\prime \prime}=x_{1}^{\prime}-\Gamma \varphi_{\sigma}^{\prime}(\sigma) \sigma^{\prime} \\
\sigma^{\prime \prime}=x_{2}^{\prime}+\left(\rho_{1}+\rho_{0}(1-u)\right) \varphi_{\sigma}^{\prime}(\sigma) \sigma^{\prime}-\alpha_{1} u \sigma^{\prime} .
\end{array}\right.
$$

С использованием системы (6), для третьей производная получим равенства

$$
\left\{\begin{array}{l}
x_{1}^{\prime \prime \prime}=-\alpha_{1} x_{1}^{\prime \prime}-\beta_{1} x_{2}^{\prime \prime}+\nu\left(\varphi_{\sigma}^{\prime \prime}(\sigma) \sigma^{2}+\varphi_{\sigma}^{\prime}(\sigma) \sigma^{\prime \prime}\right) \\
x_{2}^{\prime \prime \prime}=x_{1}^{\prime \prime}-\Gamma\left(\varphi_{\sigma}^{\prime \prime}(\sigma) \sigma^{\prime 2}+\varphi_{\sigma}^{\prime}(\sigma) \sigma^{\prime \prime}\right) \\
\sigma^{\prime \prime \prime}=x_{2}^{\prime \prime}+\left(\rho_{1}+\rho_{0}(1-u)\right)\left(\varphi_{\sigma}^{\prime \prime}(\sigma) \sigma^{\prime 2}+\varphi_{\sigma}^{\prime}(\sigma) \sigma^{\prime \prime}\right)-\alpha_{1} u \sigma^{\prime \prime}
\end{array}\right.
$$

Для нахождения зависимости кривизны цикла системы (1) от ее координат рассмотрим расширенную систему вида

$$
\left\{\begin{array}{l}
\dot{x}=A x+b \varphi(\sigma) \\
\dot{\sigma}=c^{T} x+\rho_{1} \varphi(\sigma)+\rho_{0}(1-u) \varphi(\sigma)-\alpha_{1} u \sigma \\
\dot{K}(t)=\psi(\sigma, x)
\end{array}\right.
$$

где $\dot{K}(t)=\frac{g^{\prime} f-3 g f^{\prime}}{2 g^{1 / 2} f^{5 / 2}}$ - производная кривизны цикла системы $(1)$, а функции $g(x ; \sigma), g^{\prime}(x ; \sigma), f(x ; \sigma)$ и $f^{\prime}(x ; \sigma)$ определяются соотношениями

$$
\begin{aligned}
& g(x ; \sigma)=\left(x_{1}^{\prime} x_{2}^{\prime \prime}-x_{1}^{\prime \prime} x_{2}^{\prime}\right)^{2}+\left(x_{2}^{\prime} \sigma^{\prime \prime}-x_{2}^{\prime \prime} \sigma^{\prime}\right)^{2}+\left(\sigma^{\prime} x_{1}^{\prime \prime}-\sigma^{\prime \prime} x_{1}^{\prime}\right)^{2}, \\
& g^{\prime}(x ; \sigma)=2\left(x_{1}^{\prime} x_{2}^{\prime \prime}-x_{1}^{\prime \prime} x_{2}^{\prime}\right)\left(x_{1}^{\prime} x_{2}^{\prime \prime \prime}-x_{1}^{\prime \prime \prime} x_{2}^{\prime}\right)+2\left(x_{2}^{\prime} \sigma^{\prime \prime}-x_{2}^{\prime \prime} \sigma^{\prime}\right)\left(x_{2}^{\prime} \sigma^{\prime \prime \prime}-x_{2}^{\prime \prime \prime} \sigma^{\prime}\right)+ \\
& +2\left(x_{1}^{\prime \prime} \sigma^{\prime}-x_{1}^{\prime} \sigma^{\prime \prime}\right)\left(x_{1}^{\prime \prime \prime} \sigma^{\prime}-x_{1}^{\prime} \sigma^{\prime \prime \prime}\right), \\
& f(x ; \sigma)=x_{1}^{\prime 2}+x_{2}^{\prime 2}+\sigma^{\prime 2}, \quad f^{\prime}(x ; \sigma)=2 x_{1}^{\prime} x_{1}^{\prime \prime}+2 x_{2}^{\prime} x_{2}^{\prime \prime}+2 \sigma^{\prime} \sigma^{\prime \prime} .
\end{aligned}
$$


Рассмотрим систему (1) в случае $\varphi(\sigma)=-\gamma+\sin (\sigma+\arcsin \gamma), \varphi(0)=0$. Основываясь на исследованиях, проведенных в работе [7], показывается, что при $\alpha_{1}=0,01, \beta_{1}=1,98, \Gamma=1,2$, $\nu=-2,26, \gamma=0,1, u=0,918, \rho_{1}=\nu_{1} \beta_{1}^{-1}=1,14, \rho_{0}=0,02$ система (1) имеет пять колебательных циклов, где $z_{1}(t), z_{2}(t), z_{3}(t)$ - устойчивые, а $z_{4}^{-}(t)$ и $z_{5}^{-}(t)$ - неустойчивые циклы. При $u=0$ система (1) становится фазовой системой, у которой также существует пять колебательных циклов $z_{10}(t), z_{20}(t), z_{30}(t)$ - устойчивые, а $z_{40}^{-}(t)$ и $z_{50}^{-}(t)$ - неустойчивые циклы. Основные характеристики всех колебательных циклов систем (1) и (2) приведены в таблицах 1 и 2 , где $z(t)$ - цикл системы, $x_{1}, x_{2}, \sigma$ - начальные условия цикла, $T_{z}$ - период цикла, $\langle K(t)\rangle$ - среднее значение кривизны цикла на периоде.

\begin{tabular}{|c|c|c|c|c|c|}
\hline \multicolumn{6}{|c|}{ Циклы нефазовой системы $(1) u=0,918, \sigma=0$} \\
\hline$z(t)$ & $z_{1}(t)$ & $z_{2}(t)$ & $z_{3}(t)$ & $z_{4}^{-}(t)$ & $z_{5}^{-}(t)$ \\
\hline$x_{1}$ & $-0,047922$ & $-0,04565$ & $-0,0505$ & $-0,02294$ & $-0,03301$ \\
\hline$x_{2}$ & 6,207151 & 14,5849 & 23,0726 & 10,02259 & 19,16191 \\
\hline$T_{z}$ & 4,5831 & 4,4728 & 4,4609 & 4,494987529 & 4,465325155 \\
\hline$\langle K(t)\rangle$ & 0,1552 & 0,0593 & 0,0369 & 0,0849 & 0,0441 \\
\hline
\end{tabular}

Таблица 1. Циклы нефазовой системы.

\begin{tabular}{|c|c|c|c|c|c|}
\hline \multicolumn{7}{|c|}{ Циклы фазовой системы $(2) u=0, \sigma=0$} \\
\hline$z(t)$ & $z_{10}(t)$ & $z_{20}(t)$ & $z_{30}(t)$ & $z_{40}^{-}(t)$ & $z_{50}^{-}(t)$ \\
\hline$x_{1}$ & $-0,866482$ & $-1,2813$ & $-1,6773$ & 0,339472 & 0,52238 \\
\hline$x_{2}$ & 5,913351 & 14,3064 & 22,7464 & 9,911372 & 19,06786 \\
\hline$T_{z}$ & 4,5866 & 4,4741 & 4,4618 & 4,49677402 & 4,46586352 \\
\hline$\langle K(t)\rangle$ & 0,1513 & 0,0582 & 0,0363 & 0,0831 & 0,0432 \\
\hline
\end{tabular}

Таблица 2. Циклы фазовой системы.

На рисунке 1 представлены проекции циклов $z_{10}(t), z_{20}(t), z_{30}(t)$ фазовой системы $(2)$ на плоскость $\left(x_{2} ; \sigma\right)$. На рисунке 2 в пространстве $\left(x_{1} ; x_{2} ; K(t)\right)$ изображены линии $L_{1}, L_{2}, L_{3}$, определяемые решениями системы (8), с начальными условиями циклов $z_{10}(t), z_{20}(t), z_{30}(t)$ системы $(2)$.

На рисунке 3 изображены линии $L_{z 10}^{1}$ и $L_{z 1}^{1}$, показывающие зависимость кривизны $K(t)$ циклов $z_{10}(t)$ и $z_{1}(t)$ фазовой и нефазовой систем от координаты $x_{1}(t)$. На рисунках 4 и 5 представлены зависимости кривизны от координат $x_{2}(t)$ и $\sigma(t)$ (Линии: $L_{z 10}^{2}, L_{z 1}^{2}$ и $L_{z 10}^{3}, L_{z 1}^{3}$ ).

На рисунке 6 - зависимость кривизны $K(t)$ циклов $z_{10}(t)$ и $z_{1}(t)$ от времени на периоде.

На рисунках 7 и 8 определены зависимости кривизны от координаты $x_{1}(t)$ соответственно для циклов $z_{20}(t), z_{2}(t)$ и $z_{30}(t), z_{3}(t)$.

Корреляционный анализ метрических характеристик циклов систем (1), (2), (8) позволяет сделать вывод о том, что отличия характеристик кривизны циклов фазовой системы (2) и нефазовой системы (1) не являются значимыми [7]. Использование понятия кривизны цикла позволяет проводить анализ близости циклов фазовой и нефазовой систем и сравнительный анализ частотноамплитудных характеристик кривизны полученных циклов [7].

Рассмотрим устойчивый цикл $z_{10}(t)$, для которого выполняются условия $z_{10}(0)=\operatorname{colon}\left(x_{11}\right.$, $\left.x_{12}, 0\right), x_{11}=-0,866, x_{12}=5,913, T_{1}=4,5866-$ период цикла $z_{10}(t)$. Для цикла $z_{10}(t)$ определим окрестность $\Omega_{10}=\left\{(x, t):\left(x_{1}-x_{11}\right)^{2}+\left(x_{2}-x_{12}\right)^{2}=R^{2}, t=0, R=1 / 2\right\}$. Используя оператор сдвига по траекториям системы (8), найдем отображение множества $\Omega_{10}$ через время $T_{1}$ в координатах $\left(x_{1}, K, t\right)$. С использованием $\Omega_{10}$ численными методами определим кривизну $K\left(x_{1}, x_{2}, \sigma\right)$ на границе множества $\Omega_{10}$ и построим множество

$$
\Omega_{x_{1}, k, 0}=\left\{\left(x_{1}, K, t\right): t=0, K=K\left(x_{1}, x_{2}, \sigma_{0}\right), \sigma_{0}(0)=0, x=\operatorname{colon}\left(x_{1}, x_{2}\right) \in \Omega_{10}\right\} .
$$




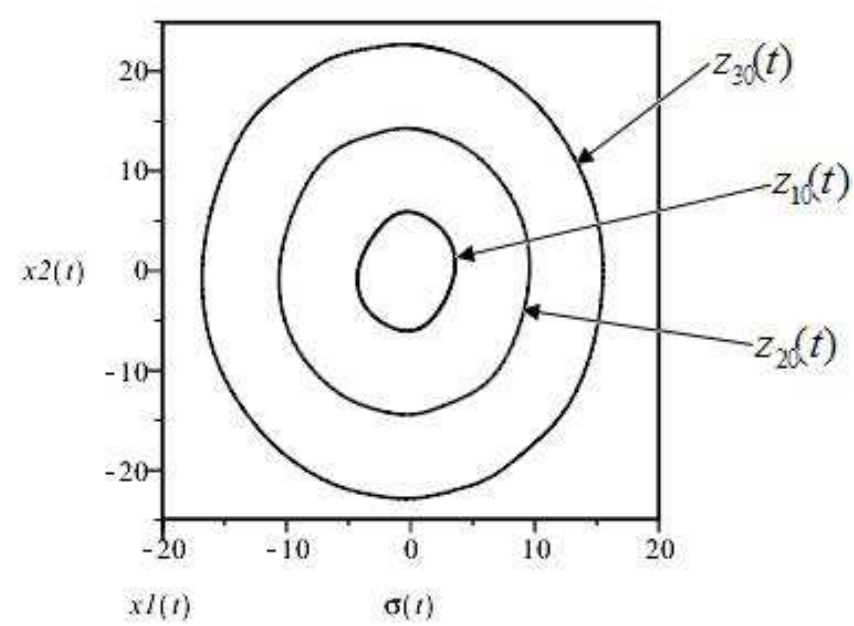

Рис. 1

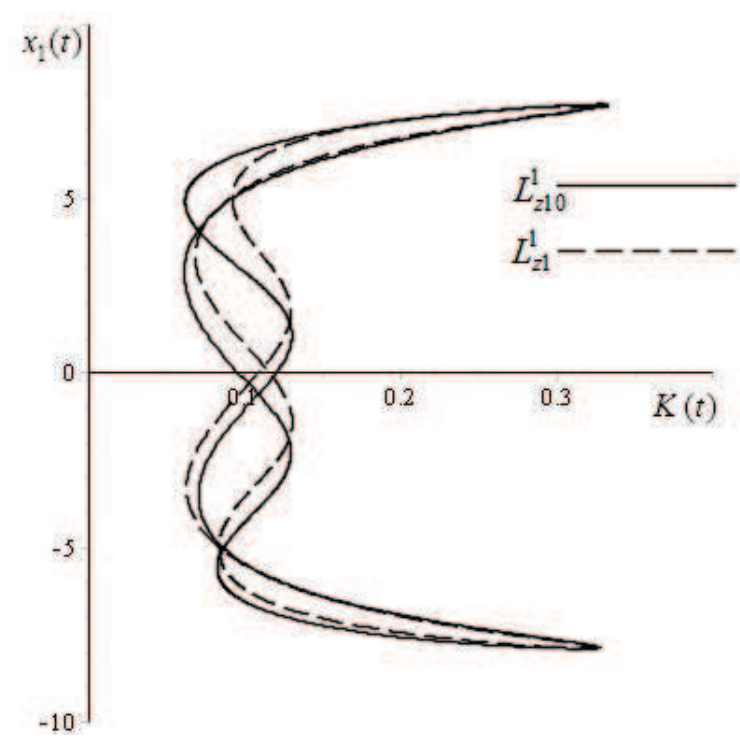

Рис. 3

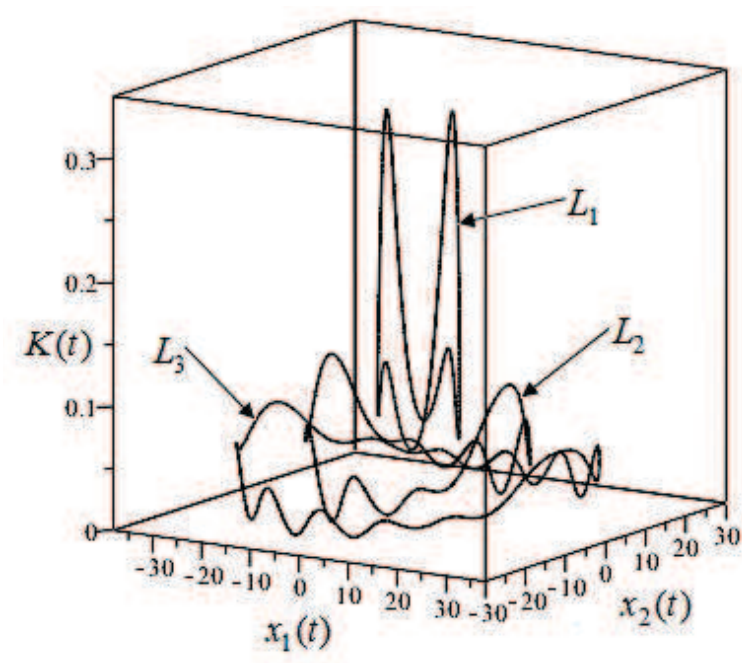

Рис. 2

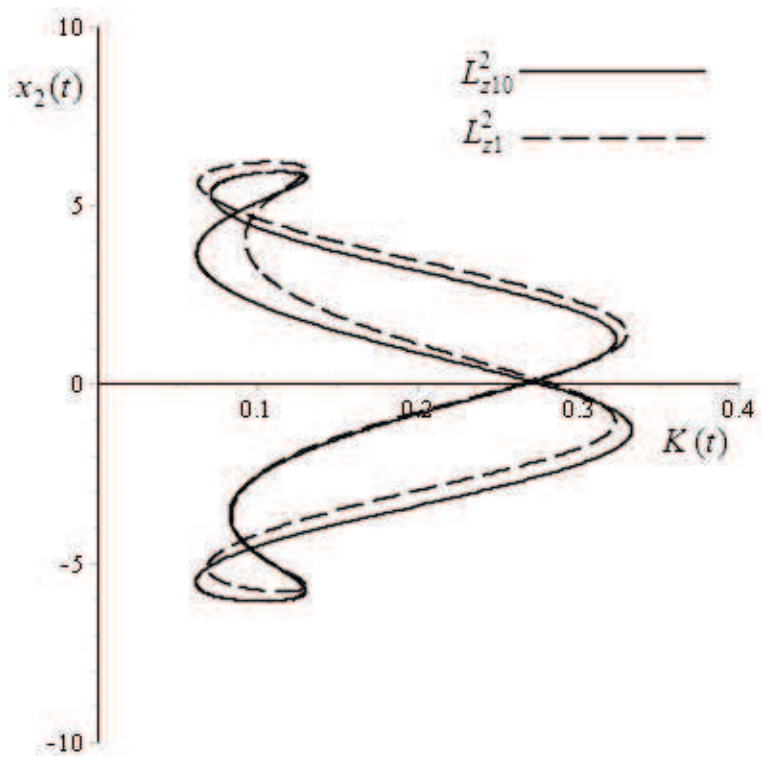

Рис. 4

Пусть $U_{x_{1}, k, 0}=P_{T_{1}} \circ T_{x_{1}, k}$, где $T_{x_{1}, k}$-оператор сдвига по траекториям системы (8), $P_{T_{1}}-$ параллельный перенос,

$$
P_{T_{1}}\left(\begin{array}{c}
x_{1} \\
K \\
t
\end{array}\right)=\left(\begin{array}{c}
x_{1} \\
K \\
t-T_{1}
\end{array}\right), \quad U_{x_{1}, k, 0}\left(\Omega_{x_{1}, k, 0}\right)=\Omega_{x_{1}, k, T_{1}} .
$$

Обозначим через $Q_{x_{1}, k, 0}\left(x_{1}, K\right)$ векторное поле оператора $U_{x_{1}, k, 0}$ на границе $\partial \Omega_{x_{1}, k, 0}$ множества $\Omega_{x_{1}, k, 0}$.

Численно показывается, что вращение векторного поля $Q_{x_{1}, k, 0}$ на границе $\partial \Omega_{x_{1}, k, 0}$ множества $\Omega_{x_{1}, k, 0}$ отлично от нуля, $\gamma\left(Q_{x_{1}, k, 0}, \partial \Omega_{x_{1}, k, 0}\right) \neq 0$. Отображение $U_{x_{1}, k, 0}$ содержит неподвижную точку оператора, определяющую начальные условия цикла первого рода фазовой системы (8). 


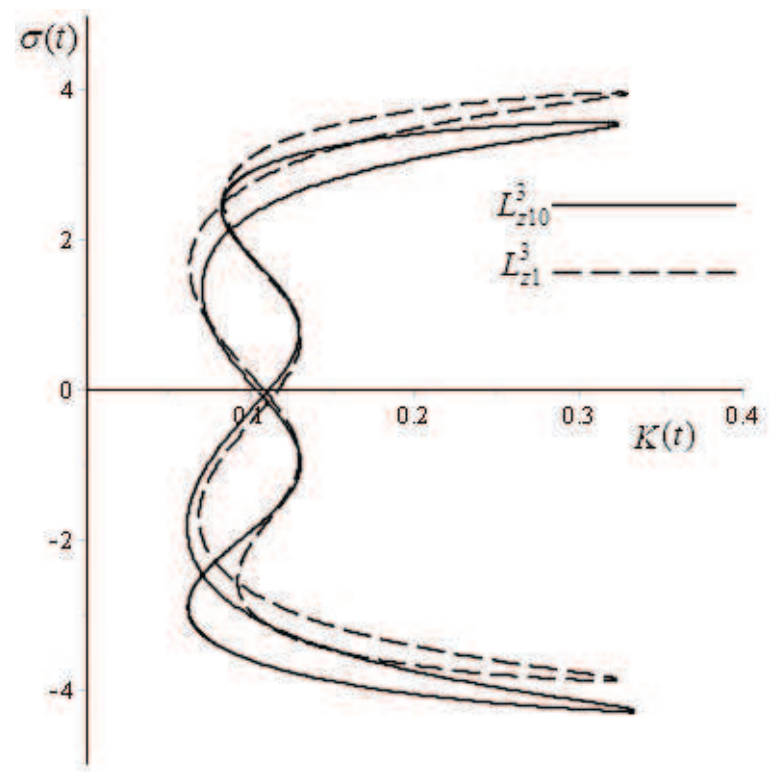

Рис. 5

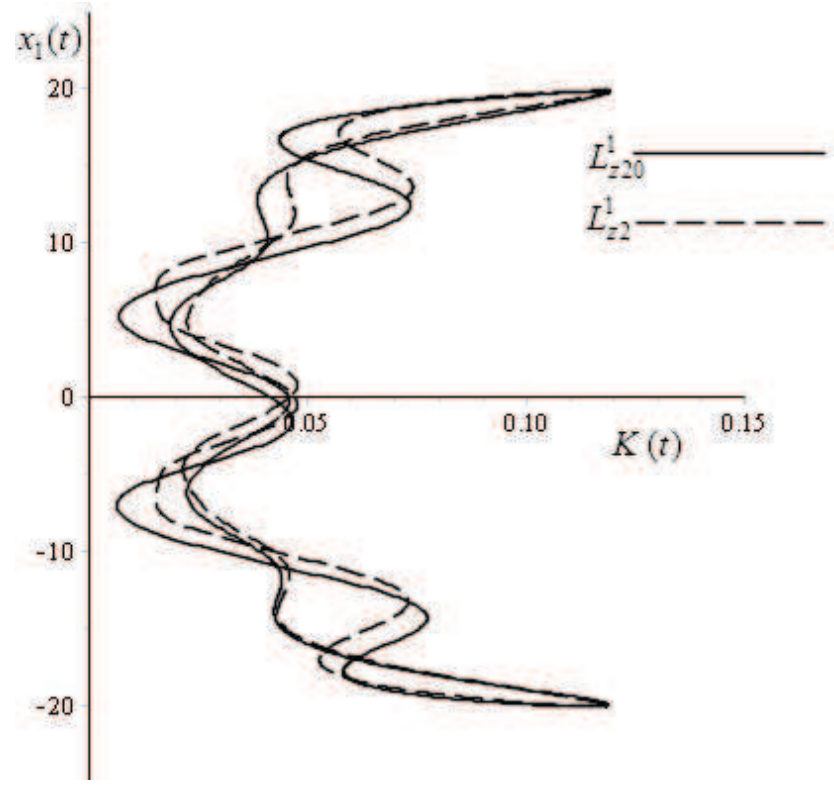

Рис. 7

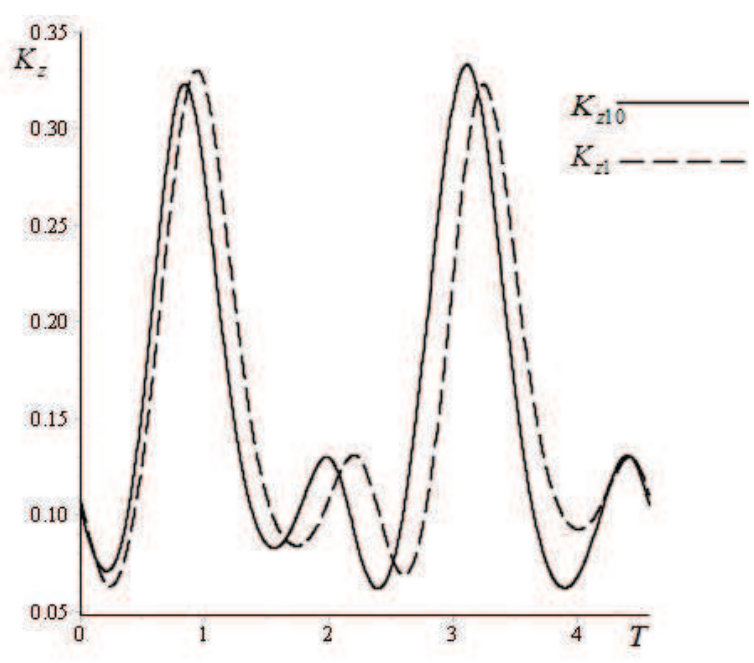

Рис. 6

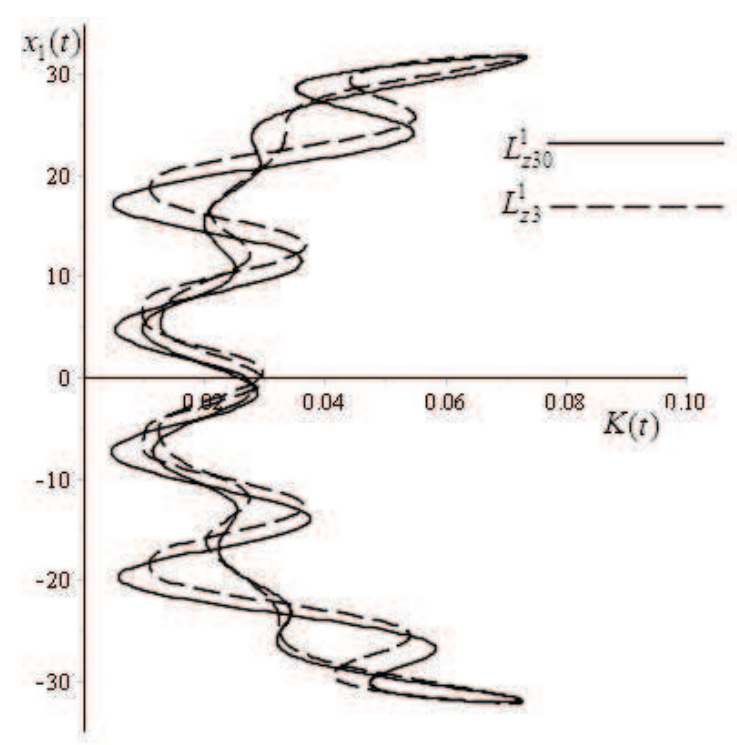

Рис. 8

Для дальнейших исследований кривизны цикла $z_{10}(t)$ переместим центр множества $\Omega_{x_{1}, k, 0}$ в начало координат. Обозначим через $P_{z_{10}}$ параллельный перенос,

$$
P_{z_{10}}\left(\begin{array}{c}
x_{1} \\
K \\
t
\end{array}\right)=\left(\begin{array}{c}
x_{1}-x_{11} \\
K-K_{10} \\
t
\end{array}\right)
$$

$K_{10}=0,1035$ - кривизна цикла $z_{10}(t)$ в момент времени $t=0, P_{z_{10}}\left(\Omega_{x_{1}, k, 0}\right)=\bar{\Omega}_{x_{1}, k, 0}$, $P_{z_{10}}\left(\Omega_{x_{1}, k, T_{1}}\right)=\bar{\Omega}_{x_{1}, k, T_{1}}$. Аналогично $\Omega_{x_{1}, k, 0}$ находится множество

$$
\Omega_{x_{2}, k, 0}=\left\{\left(x_{2}, K, t\right): t=0, K=K\left(x_{1}, x_{2}, \sigma_{0}\right), \sigma_{0}(0)=0, x=\operatorname{colon}\left(x_{1}, x_{2}\right) \in \Omega_{10}\right\},
$$




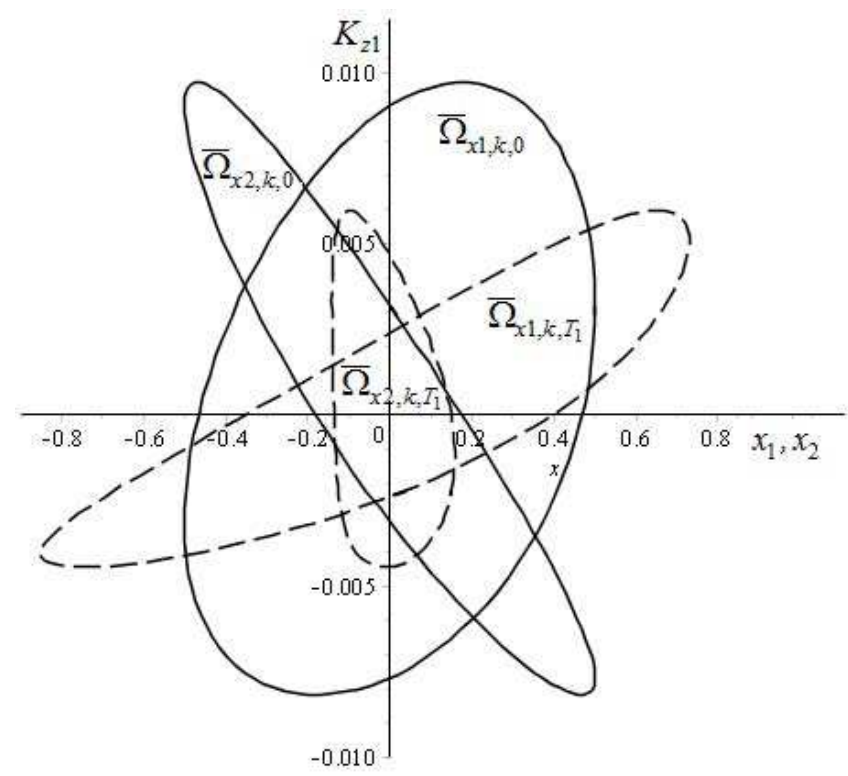

Рис. 9

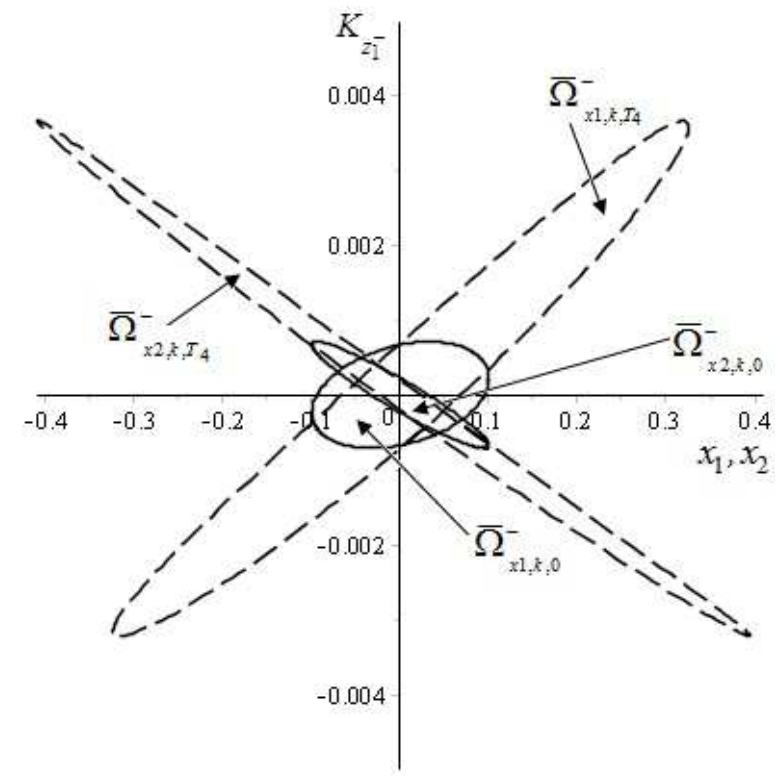

Рис. 10

которое является отображением множества $\Omega_{10}$ оператором сдвига по траекториям системы (8) через время $T_{1}$.

Пусть $U_{x_{2}, k, 0}=P_{T_{1}} \circ T_{x_{2}, k}$, где $T_{x_{2}, k}$-оператор сдвига по траекториям системы (8), $P_{T_{1}}-$ параллельный перенос, тогда $U_{x_{2}, k, 0}\left(\Omega_{x_{2}, k, 0}\right)=\Omega_{x_{2}, k, T_{1}}, \bar{P}_{z_{10}}\left(\Omega_{x_{2}, k, 0}\right)=\bar{\Omega}_{x_{2}, k, 0}, \bar{P}_{z_{10}}\left(\Omega_{x_{2}, k, T_{1}}\right)=$ $\bar{\Omega}_{x_{2}, k, T_{1}}, \bar{P}_{z_{10}}$ - параллельный перенос,

$$
\bar{P}_{z_{10}}\left(\begin{array}{c}
x_{2} \\
K \\
t
\end{array}\right)=\left(\begin{array}{c}
x_{2}-x_{12} \\
K-K_{10} \\
t
\end{array}\right) .
$$

Для геометрической интерпретации полученных множеств выберем систему координат с совмещенными осями $O X_{1}, O X_{2}$ и вертикальной осью $O K_{z}$ для кривизны. На рис. 9 изображены множества $\bar{\Omega}_{x_{1}, k, 0}, \bar{\Omega}_{x_{1}, k, T_{1}}, \bar{\Omega}_{x_{2}, k, 0}, \bar{\Omega}_{x_{2}, k, T_{1}}$. Численными методами показывается, что $\bar{\Omega}_{x_{1}, k, T_{1}} \cap \bar{\Omega}_{x_{2}, k, T_{1}} \subset \bar{\Omega}_{x_{1}, k, 0}$. Предложенные отображения $U_{x_{1}, k, 0}, U_{x_{2}, k, 0}$ дают возможность их использования для численного определения начальных условий и периода цикла системы (8) [6].

На рис. 10 представлены множества $\bar{\Omega}_{x_{1}, k, 0}^{-} \bar{\Omega}_{x_{1}, k, T_{4}}^{-}, \bar{\Omega}_{x_{2}, k, 0}^{-}, \bar{\Omega}_{x_{2}, k, T_{4}}^{-}$полученные для неустойчивого цикла $z_{40}^{-}$, и множества $\Omega_{40}=\left\{(x, t):\left(x_{1}-x_{41}\right)^{2}+\left(x_{2}-x_{42}\right)^{2}=R^{2}, t=0\right\}$ где $R=0,1$, а $x_{41}, x_{42}$ - начальные условия неустойчивого цикла $z_{40}^{-}$. Численными методами показываются включения $\bar{\Omega}_{x_{1}, k, T_{4}}^{-} \cap \bar{\Omega}_{x_{2}, k, T_{4}}^{-} \subset \bar{\Omega}_{x_{1}, k, 0}^{-}, \bar{\Omega}_{x_{1}, k, T_{4}}^{-} \cap \bar{\Omega}_{x_{2}, k, T_{4}}^{-} \subset \bar{\Omega}_{x_{2}, k, 0}^{-}$, это дает возможность для неустойчивого цикла определить отображения переводящие окрестности цикла в себя, что позволяет предложить численный подход определения начальных условий неустойчивого цикла [4-6].

Включение в систему (2) уравнения, содержащего кривизну, дает возможность использовать характеристики устойчивости кривизны циклов в качестве критерия выбора режимов скрытой синхронизации для системы фазовой автоподстройки частоты [7]. Для определения характеристик устойчивости кривизны предлагается использовать показатели Ляпунова [8].

$$
\lambda_{N}=\frac{1}{t_{N}-t_{0}} \sum_{i=1}^{N} \log _{2} \frac{d\left(\Omega_{x_{1}, k, t_{i}}\right)}{d\left(\Omega_{x_{1}, k, t_{i-1}}\right)},
$$

где $d\left(\Omega_{x_{1}, k, t_{i}}\right)$ - площадь множества $\Omega_{x_{1}, k, t_{i}}, d\left(\Omega_{x_{1}, k, t_{i-1}}\right)$ - площадь множества $\Omega_{x_{1}, k, t_{i-1}}$, множество $\Omega_{x_{1}, k, t_{i}}$ является отображением множества $\Omega_{x_{1}, k, t_{i-1}}$ оператором сдвига $T_{x_{1}, k}$ по траекториям системы $(8)$ в координатах $\left(x_{1}, K, t\right)$ через время $\Delta t_{i}=t_{i}-t_{i-1}$. Найдем для устойчивого цикла 
$z_{10}(t)$ показатели Ляпунова в координатах $\left(x_{1}, K, t\right)$, в качестве $t_{i}$ возьмем $t_{i}=(i-1) T_{1}, i=\overline{1,7}$, $T_{1}=4,5866-$ период цикла $z_{10}(t)$. Численными методами найдем площади множеств $\Omega_{x_{1}, k, t_{i}}$, $i=\overline{1,7}$. Значения $\lambda_{N}$, полученные с использованием (9), содержатся в таблице 3. Аналогично для цикла $z_{10}(t)$, численными методами найдем площади множеств $\Omega_{x_{2}, k, t_{i}}, i=\overline{1,7}$ в координатах $\left(x_{2}, K, t\right)$, полученные значения содержатся в таблице 4.

В таблицах 3, 4 приведены значения показателей Ляпунова для всех устойчивых циклов фазовой системы $(2)$, с использованием окрестностей циклов в координатах $\left(x_{1}, K, t\right),\left(x_{2}, K, t\right)$.

\begin{tabular}{|c|c|c|c|c|c|c|c|}
\hline \multirow{2}{*}{$z_{i}(t)$} & \multirow{2}{*}{$x_{1}(t), K_{z_{i}}(t)$} & \multicolumn{6}{|c|}{$\lambda_{i}$} \\
\cline { 3 - 8 } & $\lambda_{1}$ & $\lambda_{2}$ & $\lambda_{3}$ & $\lambda_{4}$ & $\lambda_{5}$ & $\lambda_{6}$ \\
\hline$z_{10}(t)$ & $x_{1}(t), K_{z_{1}}(t)$ & $-0,3211$ & $-0,1128$ & $-0,0747$ & $-0,0577$ & $-0,0476$ & $-0,0410$ \\
\hline$z_{20}(t)$ & $x_{1}(t), K_{z_{2}}(t)$ & $-0,8026$ & $-0,2871$ & $-0,1554$ & $-0,1113$ & $-0,0885$ & $-0,0742$ \\
\hline$z_{30}(t)$ & $x_{1}(t), K_{z_{3}}(t)$ & $-0,4754$ & $-0,3934$ & $-0,1647$ & $-0,1062$ & $-0,0296$ & $-0,0657$ \\
\hline
\end{tabular}

Таблица 3

\begin{tabular}{|c|c|c|c|c|c|c|c|}
\hline \multirow{2}{*}{$z_{i}(t)$} & \multirow{2}{*}{$x_{2}(t), K_{z_{i}}(t)$} & \multicolumn{6}{|c|}{$\lambda_{i}$} \\
\cline { 3 - 8 } & $\lambda_{1}$ & $\lambda_{2}$ & $\lambda_{3}$ & $\lambda_{4}$ & $\lambda_{5}$ & $\lambda_{6}$ \\
\hline$z_{10}(t)$ & $x_{2}(t), K_{z_{1}}(t)$ & $-0,3209$ & $-0,1130$ & $-0,0749$ & $-0,0579$ & $-0,0478$ & $-0,0411$ \\
\hline$z_{20}(t)$ & $x_{2}(t), K_{z_{2}}(t)$ & $-0,8024$ & $-0,2871$ & $-0,1555$ & $-0,1114$ & $-0,0886$ & $-0,0743$ \\
\hline$z_{30}(t)$ & $x_{2}(t), K_{z_{3}}(t)$ & $-0,4753$ & $-0,3932$ & $-0,1646$ & $-0,1061$ & $-0,0802$ & $-0,0656$ \\
\hline
\end{tabular}

Таблица 4

Показатели Ляпунова для устойчивых циклов являются отрицательными величинами, при этом наименьшее значение $\lambda_{6}=-0,0743$ определено для устойчивого цикла $z_{20}(t)$ в координатах $\left(x_{2}, K, t\right)$. Полученные результаты позволяют использовать показатели Ляпунова в качестве критерия качества режимов скрытой синхронизации для систем фазовой автоподстройки частоты.

3. Заключение. В работе предложено расширение исходной системы дифференциальных уравнений за счет включения в нее уравнения производной кривизны решения. Расширение исходной системы дифференциальных уравнений позволило в новом фазовом пространстве получить периодические решения одной из координат, которой является кривизна. С использованием понятия кривизны цикла проведен анализ близости циклов фазовой и нефазовой систем. Предложено использование вращения векторного поля расширенной системы для определения начальных значений и периодов устойчивых и неустойчивых циклов. Определены показатели Ляпунова для кривизны циклов в случае мультистабильности. Практическая значимость полученных результатов заключается в использовании кривизны при выборе режимов скрытой синхронизации в случае мультистабильности.

\section{СПИСОК ЛИТЕРАТУРЫ}

1. Абрамов В. В. Условия существования периодического решения дифференциального уравнения второго порядка с квадратичной нелинейной частью// Вестн. РАЕН. - 2018. - 18, № 4. - С. 3-7.

2. Леонов Г. А., Буркин И. М., Шепелявый А. И. Частотные методы в теории колебаний. - СПб.: Изд-во СПбГУ, 1992.

3. Лискина Е. Ю. Условия существования ненулевых периодических решений нелинейной автономной динамической системы второго порядка в случае пары нулевых собственных значений матрицы системы линейного приближения // Вестн. РАЕН. - 2017. - 17, № 4. - С. 38-43.

4. Мамонов C. С., Харламова А. О. Вынужденная синхронизация систем фазовой автоподстройки с запаздыванием// Вестн. Рязан. гос. радиотехн. ун-та. - 2017. - 62. - С. 26-35. 
5. Мамонов C. C., Харламова A. О. Определение условий существоваия предельных циклов первого рода систем с цилиндрическим фазовым пространством// Ж. Средневолж. мат. о-ва. - 2017. - 19, № 1. C. $67-76$.

6. Мамонов С. С., Харламова А. О., Ионова И. В. Колебательно-вращательные циклы фазовой системы дифференциальных уравнений// Вестн. РАЕН. - 2018. - 18, № 4. - С. 51-57.

7. Мамонов С. С., Харламова А. О., Ионова И. В. Кривизна колебательных циклов фазовых систем// Вестн. РАЕН. - 2019. - 19, № 2. - С. 105-110.

8. Мун Ф. Хаотические колебания. - М.: Мир, 1990.

Мамонов Сергей Станиславович

Рязанский государственный университет имени С. А. Есенина

E-mail: s.mamonov@365.rsu.edu.ru

Ионова Ирина Викторовна

Рязанский государственный университет имени С. А. Есенина

E-mail: i.ionova@365.rsu.edu.ru

Харламова Анастасия Олеговна

Рязанский государственный университет имени С. А. Есенина

E-mail: a.harlamova@365.rsu.edu.ru 